\title{
Current Status of Ovarian Cancer Screening and Diagnosis
}

Ryan W.R. Guilbault, Morgan C. Smith, Priya J. Desai and Jaehwa Choi*

Mercer University School of Medicine, Columbus, GA, USA

“Corresponding author: Jaehwa Choi, 1501 Mercer University Drive, Macon, GA, 31207, USA, Tel: (478) 301- 2556; E-mail: Choi_j@med.mercer.edu

Received: April 27, 2018; Accepted: May 14, 2018; Published: May 21, 2018

\section{Retraction Note}

The article entitled "Current Status of Ovarian Cancer Screening and Diagnosis" has been accepted for publication in the Journal of Bioanalysis \& Biomedicine considering the statements provided in the article as personal opinion of the author which was found not having any conflict or biasness towards anything. As the article was a perspective one, information provided by the author was considered as an opinion to be expressed through publication.

Publisher took decision to make the article online solely based on the reviewers suggestion which considered the article not but a personal opinion of the author. However, it is found that the author have some personal concerns and issues, therefore, being retracted from the journal. 